\title{
Influence de la modification de la charge des micelles de caséine sur le taux de caséinomacropeptide libéré par la présure au moment de la coagulation du lait
}

\author{
par
}

A. PIERRE

\section{R és u m é}

Les résultats exposés montrent que la quantité de caséinomacropeptide (CMP) libéré par la présure au moment de la coagulation du lait dépend du $\mathrm{pH}$. On a observé la coagulation après la libération de $43 \%$ du CMP à pH 5,0 et de $100 \%$ du CMP à pH 7,2, valeurs extrêmes de $\mathrm{pH}$ étudiées. Une préacidification du lait à $\mathrm{pH} 5,0$, suivie d'un réajustement du $\mathrm{pH}$ dans les mêmes conditions que dans l'expérience précédente ne modifie pas les résultats que l'on obtient. Par contre, une addition de lysozyme au lait abaisse le taux de libération du CMP observé à la coagulation. L'effet du lysozyme augmente avec sa concentration mais varie avec le $\mathrm{pH}$. Son efficacité est maximum aux $\mathrm{pH}$ inférieurs à 6,0 et diminue quand le $\mathrm{pH}$ augmente pour s'annuler à $\mathrm{pH} 7,0$.

Les différents traitements appliqués ci-dessus au lait ont une incidence sur les temps de coagulation dont les valeurs sont également étudiées.

Les résultats sont discutés, compte tenu des connaissances actuelles en matière de charge micellaire, pour leurs conséquences dans la compréhension du mécanisme de la coagulation du lait par la présure.

Mots clés : Lait - Présure - Coagulation - Caséinomacropeptide.

Institut National de la Recherche Agronomique, Laboratoire de Recherches de Technologie Laitière, 65, rue de Saint-Brieuc - 35042 Rennes cedex (France). 


\section{Su m m a ry}

INFLUENCE OF A CHANGE IN MICELLAR CHARGE ON THE PROPORTION OF CASEINOMACROPEPTIDE RELEASED BY RENNET ON COAGULATION OF MILK

The results show that the proportion of caseinomacropeptide $(C M P)$ released by rennet on clotting of milk is $p H$ dépendent. $43 \%$ of CMP is released in a milk at $p H 5.0$ when coagulation occurs and $100 \%$ in a milk at $p H$ 7.2. These $p H$ values are the highest and the lowest values tested. When a milk was preacidified at $\mathrm{pH} 5.0$ before the adjustment at the final $\mathrm{pH}$ value and then treated as in the previous experiments, the results are not modified by the pretreatment. On the other hand, a lysozyme addition to milk decreases the CMP release level to which the coagulation is observed. The lysozyme effect increases with concentration and is related to $p H$. Its efficacity is highest at $p H$ below 6.0 and decreases when $\mathrm{pH}$ increases. At $\mathrm{pH} 7.0$, there is no more lysozyme effect on CMP release level on coagulation.

The rennet clotting times obtained for milks after the treatments described are also reported.

The results are discussed for their implications in the role of the micellar charge in the mechanisms of rennet coagulation of milk.

Key words:

Milk - Rennet - Coagulation - Caseinomacropeptide.

\section{INTRODUCTION}

Les mécanismes mis en jeu lors de la coagulation du lait par la présure ne sont pas entièrement compris. Il se produit une agrégation des micelles de caséine qui étaient à l'état natif en suspension stable dans le lait. La stabilité de la micelle semble résulter de différents types d'interactions. D'une part, des forces d'attraction entre les constituants micellaires seraient responsables de la structure micellaire et de sa cohésion, d'autre part, des forces de répulsion dues à l'existence d'un potentiel de surface favoriseraient la stabilité en solution. On considère actuellement que la caséine Kappa, localisée à la périphérie de la micelle (Payens, 1966; Schmidt, 1980), serait responsable de cette charge superficielle. Elle aurait par ailleurs un rôle de " colloïde protecteur », dont la nature n'est pas encore bien définie. Le mécanisme de la stabilisation est certainement en relation avec les propriétés polaires de cette caséine (Waugh et von Hippel, 1956 ; Pyne et Mc Gann, 1962 ; Noelken, 1966), sans que l'on sache si le rôle prépondérant doit être attribué à la polarité hydrophile-hydrophobe ou à la polarité de charge. En effet, les théo- 
ries élaborées à la suite de l'étude de colloïdes lyophobes n'ont pu rendre compte de toutes les propriétés de la micelle de caséine (Payens, 1966), sans doute à cause de son hétérogénéité de composition (caséine, phosphate de calcium colloïdal).

Cependant, il est très probable que la diminution importante du potentiel observée lors de la coagulation du lait par la présure (Green et Crutchfield, 1971) soit, au moins en partie, à l'origine de la déstabilisation. On attribue cette baisse de potentiel à l'hydrolyse de la partie C terminale de la caséine Kappa, le CMP, qui est très chargé négativement. Dalgleish (1979) a montré que, dans le lait, l'hydrolyse de $88 \%$ du CMP était nécessaire pour la coagulation. Foltmann (1959) a observé que la coagulation se produisait à un taux d'hydrolyse du CMP plus faible quand le $\mathrm{pH}$ du lait était plus bas que le $\mathrm{pH}$ naturel et Green et Marshall (1977) ont fait la même observation après addition de certaines substances chargées positivement. Ces résultats semblent confirmer l'importance de la variation de charge micellaire dans le phénomène de coagulation par la présure. Une modification de la charge micellaire semble permettre la coagulation à des taux d'hydrolyse du CMP différents de ceux du lait.

Nous avons étudié la variation du taux d'hydrolyse de la caséine Kappa au moment de la coagulation par la présure, en fonction de la charge des micelles de caséine; cette charge était modifiée artificiellement en agissant sur le $\mathrm{pH}$ du lait et (ou) en ajoutant une molécule chargée (lysozyme).

\section{MATERIELS ET METHODES}

\section{Matériels}

Les échantillons de lait cru de mélange provenaient de la ferme expérimentale de l'I.N.R.A.; ils étaient écrémés par centrifugation et additionnés d'un conservateur $\left(\mathrm{NaN}_{3} \quad 0,02 \%\right)$. Le lysozyme de blanc d'œuf de poule provenait de chez Sigma Chemical Co Ltd et les cellules sèches de Micrococcus lysodeikticus de chez Difco. La présure cristallisée en poudre provenait de chez C. Hansen Ltd.

\section{Méthodes}

Le $\mathrm{pH}$ était mesuré à l'aide d'un appareil RADIOMETER PHM84. Dans certains échantillons de lait, le $\mathrm{pH}$ était modifié à l'aide de solutions de soude ou d'acide chlorhydrique jusqu'à la valeur désirée; dans le cas où le $\mathrm{pH}$ devait être abaissé à 5,0 puis remonté à des valeurs supérieures, une période de 15 min était ménagée entre les deux traitements. Nous avions préalablement étudié la cinétique de l'échange du calcium dans ces conditions opératoires et 
déterminé que lors d'une acidification, la teneur en calcium soluble se stabilisait après $120 \mathrm{~min}$, et que $90 \%$ était déjà solubilisé après $15 \mathrm{~min}$. Lors de la remontée du $\mathrm{pH}$, l'équilibre était atteint en $15 \mathrm{~min}$. La dilution maximum était de $2 \%$ et une quantité d'eau adéquate était ajoutée dans les autres flacons pour uniformiser la dilution. Dans certains échantillons, du lysozyme était ajouté directement dans les laits soumis à une forte agitation. Les laits étaient ensuite placés au bain marie à $32^{\circ} \mathrm{C}$ pendant $90 \mathrm{~min}$ et le $\mathrm{pH}$ mesuré à nouveau après ce temps.

Les tests de coagulation étaient réalisés à $32^{\circ} \mathrm{C}$. Les temps de coagulation étaient déterminés sur un volume de $3 \mathrm{ml}$ de lait auquel la présure (solution à $0,4 \%$ dans de l'eau, $\mathrm{pH}$ final 6,0 ) était ajoutée sous un volume de $100 \mu \mathrm{l}$; le tube contenant le mélange était maintenu incliné dans un bain marie et animé d'un mouvement de rotation lent (18 t.p.m.). Le moment de la coagulation était surveillé visuellement et indiqué par l'apparition de flocons de caillé dans le film de lait à la surface du tube. Au moment de la coagulation, on ajoutait au lait $12 \mathrm{ml}$ d'une solution d'acide trichloracétique à $15 \%$ ou à $2,5 \%$ selon la séparation voulue, et le tube était soumis à une très forte agitation. Après un repos de $30 \mathrm{~min}$, une filtration sur papier (Durieux filtration lente) était effectuée.

La teneur en CMP des filtrats était déterminée par HPLC, selon une méthode mise au point au Laboratoire et analogue dans son principe à celle de Van Hooydonk et Olieman (1982). La séparation était effectuée sur un appareil Varian 5000 équipé d'une colonne de gel filtration G3000 SW et d'un filtre optique à $200 \mathrm{~nm}$. L'élution était réalisée avec un tampon phosphate (phosphate de potassium dihydrogène $1,4 \mathrm{~g} / 1$ amené à $\mathrm{pH} 2.2$ avec de l'acide phosphorique concentré) à la température ambiante et avec un débit de $1 \mathrm{ml} / \mathrm{min}$. L'étalonnage a été réalisé avec du CMP purifié, obtenu à partir de l'hydrolyse par la présure de caséinate de sodium industriel (solution aqueuse à $6 \%, \mathrm{pH} 6,5)$. Après hydrolyse, le paracaséinate était précipité à $\mathrm{pH} 4,6$. Le surnageant, contenant le CMP, était concentré par ultrafiltration en présence de calcium, puis lyophilisé. La poudre obtenue contenait $50 \%$ de minéraux. Le diagramme d'élution en HPLC dans les conditions décrites montraient la présence d'un seul pic. La teneur en azote total et le dosage des acides aminés de la fraction non minérale ont montré une bonne concordance avec la composition théorique du CMP. La fraction azotée était soluble en totalité dans le TCA à $2 \%$ et $50 \%$ était soluble dans le TCA à $12 \%$. Dans le texte nous appellerons conventionnellement CMP-12\%, la fraction CMP soluble dans le TCA à $12 \%$ (qui correspond au CMP glycosylé) et la fraction soluble dans le TCA à $2 \%$ (qui correspond au CMP total), CMP $2 \%$.

Les absorptions spécifiques à $200 \mathrm{~nm}$ du CMP-12\% et du CMP-2 \% étaient identiques. 
La détermination quantitative était effectuée en mesurant les hauteurs de pics. Il était possible de détecter une teneur en CMP de $50 \mu \mathrm{g} / \mathrm{ml}$; la précision obtenue lors de mesures sucessives du même échantillon donne un coefficient de variation de $2 \%$.

Le taux de fixation du lysozyme sur les micelles était déterminé en comparant la quantité totale ajoutée au lait et la quantité retrouvée dans les surnageants de laits après centrifugation des échantillons à $78000 \mathrm{~g}$ pendant $90 \mathrm{~min}$ à $30^{\circ} \mathrm{C}$. Le lysozyme était dosé dans les surnageants par la méthode de Shugar (1952). Le dosage du calcium était effectué par spectrométrie d'absorption atomique; le calcium soluble des solutions de caséinate était dosé sur la fraction ultrafiltrable séparée par ultrafiltration sur cônes Amicon CF 25; le calcium ionique était mesuré à l'aide d'une électrode spécifique pour les ions calcium (Radiometer) étalonnée sur des solutions de chlorure de cacium, contenant $3,2 \mathrm{~g} / 1$ de chlorure de sodium pour augmenter la force ionique et se rapprocher de celle du lait.

\section{RESULTATS}

\section{Pourcentage de CMP libéré à la coagulation Effet du $\mathrm{pH}$}

Après addition de présure à des échantillons de lait dont le $\mathrm{pH}$ a été préalablement amené à des valeurs comprises entre 5,2 et 7,2 , on observe que la coagulation se produit alors que des quantités différentes du CMP ont été libérées (fig. 1 a). A pH 5,2 la coagulation commence au moment où $43 \%$ du CMP- $2 \%$ sont libérés. Le taux d'hydrolyse de la caséine Kappa au moment de la coagulation augmente avec le $\mathrm{pH}$ du lait; au $\mathrm{pH}$ naturel du lait, 6,7, il est de $92 \%$; au $\mathrm{pH}$ le plus élevé qui a été étudié, 7,2, il est de $100 \%$.

A chaque $\mathrm{pH}$ l'action hydrolytique de la présure se poursuit après la coagulation jusqu'à ce que tout le CMP ait été libéré. La teneur totale en CMP ( $2 \%$ et $12 \%$ ) reste alors constante pendant une durée d'incubation égale à 6 fois le temps de coagulation. Les pourcentages ont été établis en prenant comme référence la teneur totale en CMP hydrolysable, qui a été évaluée dans les échantillons de lait à $\mathrm{pH}$ 6,7 après une durée d'incubation en présence de présure égale à 3 fois le temps de coagulation. Le pourcentage de libération du CMP glycosylé (12\%) et du CMP total (2\%) sont identiques à un $\mathrm{pH}$ donné. La proportion relative de l'un par rapport à l'autre est de $51 \%$ à tous les $\mathrm{pH}$. Aussi, dans la suite de l'expérimentation nous avons étudié seulement l'une des deux fractions, le CMP-12\%. Dans la suite du texte, le terme CMP sera donc utilisé sans préciser systématiquement s'il s'agit du CMP total ou glycosylé. 


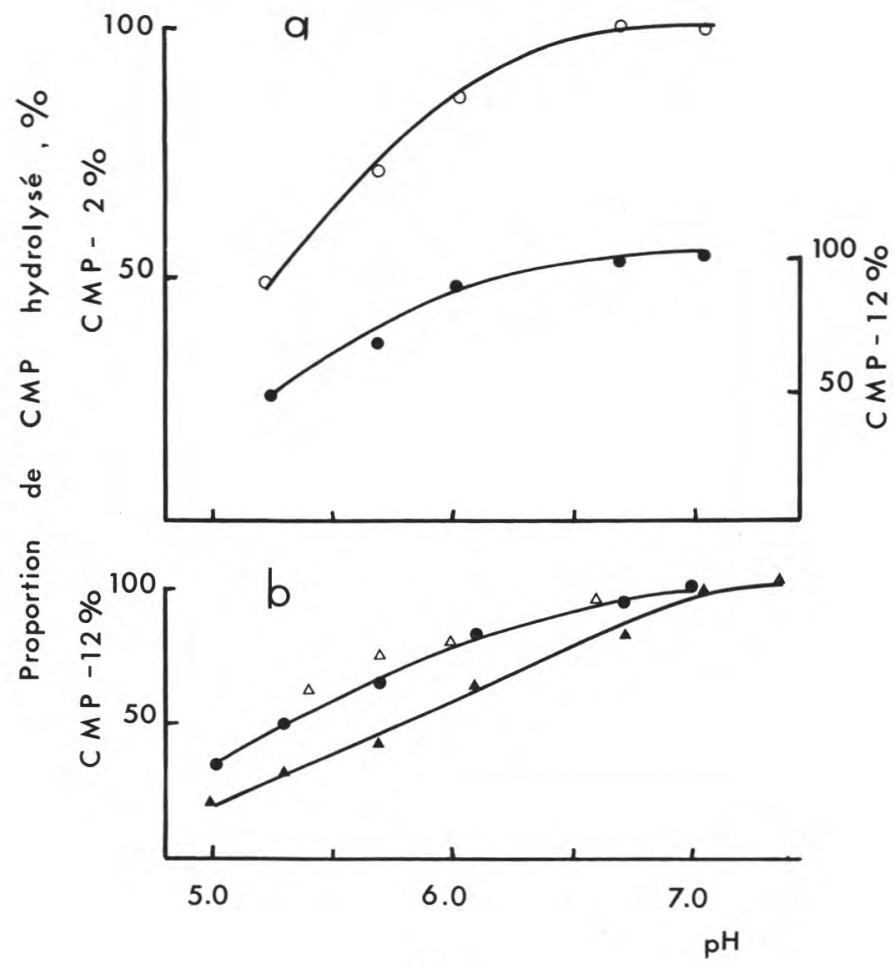

fig. 1

Proportion de CMP libéré au moment de la coagulation du lait à différents $\mathrm{pH}$

1a : CMP soluble dans le TCA à $12 \%(\bullet)$; dans le TCA à $2 \%(0)$. $1 \mathrm{~b}$ : CMP soluble dans le TCA à $12 \%$ à la coagulation d'un lait témoin $(\bullet)$, avec acidification transitoire à $\mathrm{pH} 5,0(\triangle)$, avec addition de lysozyme $(0,23 \mathrm{mM})(\boldsymbol{\Delta})$.

Proportion of hydrolysed CMP on coagulation of milk as a function of $\mathrm{pH}$

1a: (•) $12 \%$ - TCA-soluble CMP, (O) $2 \%$ TCA-soluble CMP.

1b: $12 \%$ TCA-soluble CMP on coagulation with $(\triangle)$ or with out $(\bullet)$ (reference sample) a preacidification of the milk at $\mathrm{pH} 5,0$ before adjustment at the $p H$ of coagulation; ( $\mathbf{\Lambda})$ with added lysozyme $(0,23 \mathrm{mM})$.

Dans une autre expérience, nous avons effectué une préacidification des laits jusqu'à $\mathrm{pH} 5,0$ pendant $15 \mathrm{~min}$ avant de les ramener à un $\mathrm{pH}$ plus élevé, de 5,5 à 7,2 . La relation entre $\mathrm{pH}$ et quantité de CMP-12\% libéré par la présure au moment de la coagulation n'est que peu modifiée par rapport aux laits acidifiés directement (fig. 1 b). 


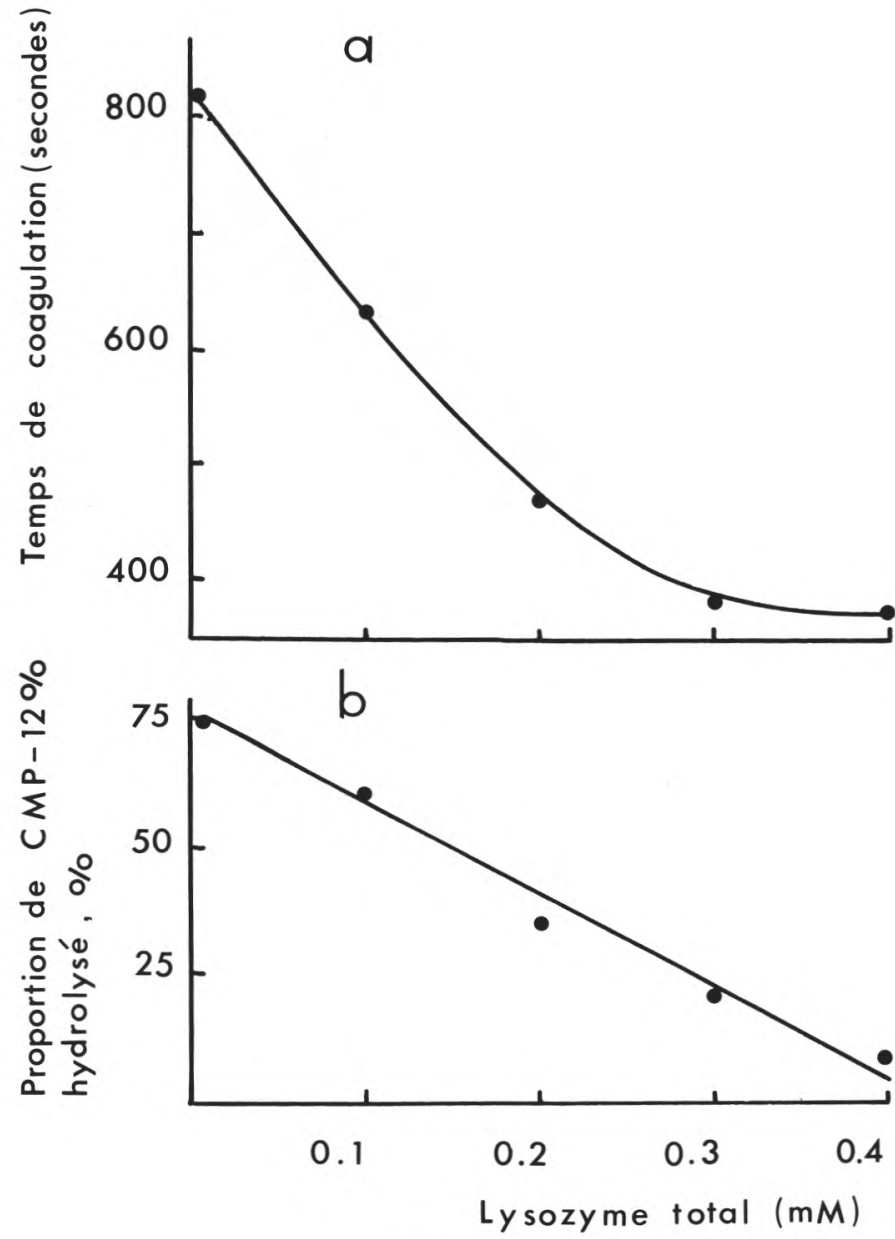

fig. 2

Influence d'une addition de lysozyme à doses croissantes dans un lait à $\mathrm{pH}$ 6,0 sur : (2-a) la proportion de CMP $12 \%$ hydrolysé au moment de la coagualtion, $(2-b)$ le temps de coagulation.

Effects of increasing amounts of added lysozyme in a milk at pH 6,0, on: (2-a) the proportion of $12 \%$ CMP hydrolysed on coagulation, $(2-b)$ the coagulation time.

\section{Effet combiné du pH et du lysozyme}

L'addition de lysozyme $(0,23 \mathrm{mM} / \mathrm{l})$ au lait après modification du $\mathrm{pH}$ entraîne une coagulation de la micelle alors que des teneurs en CMP-12\% plus faibles ont été libérées (fig. 1 b). L'effet de 


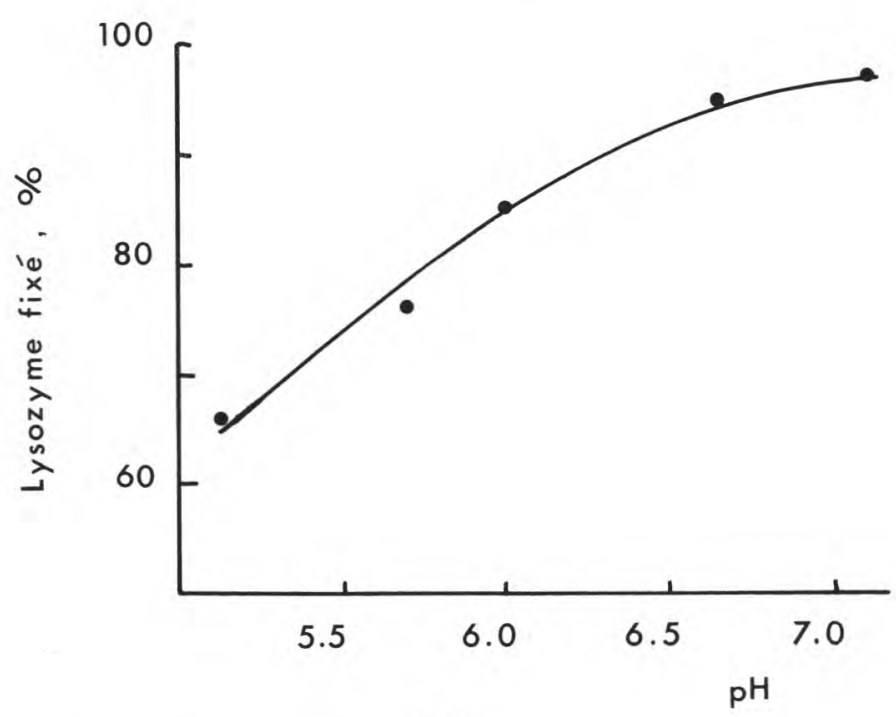

fig. 3

Influence du $\mathrm{pH}$ du lait sur la proportion de lysozyme fixé sur la micelle. La quantité totale de lysozyme ajoutée au lait était de $0,4 \mathrm{mM}$.

Effect of the $\mathrm{pH}$ of the milk on the proportion of lysozyme bound to micelles (total amount of lysozyme added 0,4 $\mathrm{mm}$ ).

l'addition d'une même quantité de lysozyme est différent selon le $\mathrm{pH}$. Aux pH inférieurs à 6,0 , la quantité de CMP-12\% libéré à la coagulation est abaissée d'une valeur constante et à peu près équivalente à $18 \%$ du CMP-12\% total. Aux pH plus élevés, la différence devient de plus en plus faible. $\mathrm{A} \mathrm{pH} \mathrm{7,0} \mathrm{et} \mathrm{au-delà,} \mathrm{le} \mathrm{lysozyme}$ n'intervient plus sur le taux de CMP libéré à la coagulation.

La figure 2-b montre que plus la quantité de lysozyme ajoutée au lait est grande, plus faible est le pourcentage de CMP-12\% libéré à la coagulation; dans cette expérience effectuée à $\mathrm{pH}$ 6,0, nous avons déterminé que $85 \%$ du lysozyme était fixé sur la micelle à toutes les concentrations en lysozyme utilisées et sédimentait avec elle lors d'une ultracentrifugation. Un résultat similaire a été rapporté par Marshall et Green (1980). La figure 3 montre que la fixation du lysozyme est fortement dépendante du $\mathrm{pH}$ de l'échantillon.

L'addition du lysozyme au lait provoque un abaissement du $\mathrm{pH}$ et une augmentation de la teneur en calcium soluble, bien que le lysozyme ajouté en soit pratiquement exempt $(0,15 \mathrm{mg}$ de $\mathrm{Ca} / \mathrm{g}$ de lysozyme). La figure 4 montre la relation entre ces deux paramètres 


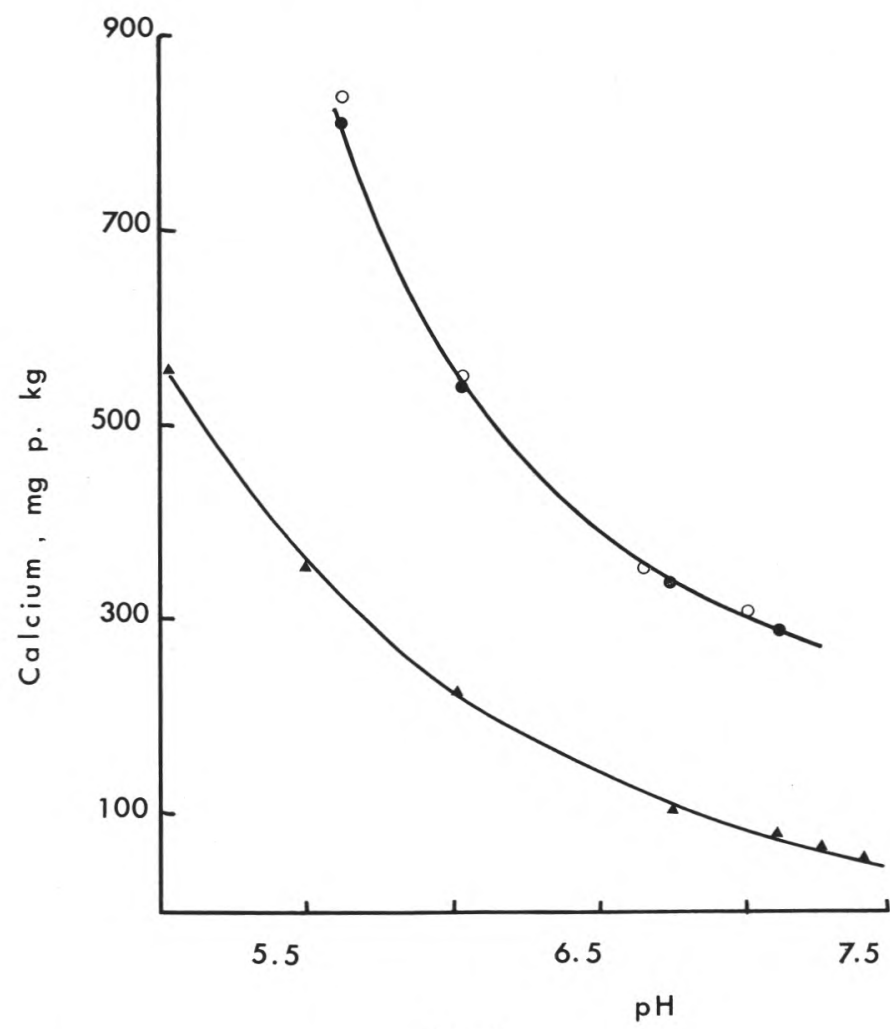

fig. 4

Influence $\mathrm{du} \mathrm{pH}$ sur la teneur en calcium ionique du lait (\$) et la teneur en calcium soluble total avant $(\bullet)$ et après $(0)$ addition de $0,4 \mathrm{mM}$ de lysozyme.

Ionic calcium level in milk ( $\mathbf{\Delta})$ and total soluble calcium before (•) and after (0) addition of lysozyme $(0,4 \mathrm{mM})$.

pour un lait avant et après addition de $0,4 \mathrm{mM} / 1$ de lysozyme. L'augmentation de teneur en calcium soluble après addition de lysozyme est de l'ordre de $20 \mathrm{mg} / \mathrm{kg}$.

\section{Incidence de ces traitements sur les temps de coagulation}

Les temps de coagulation mesurés dans les laits au cours des expériences précédentes sont reportés dans le tableau 1. L'acidification préalable du lait à $\mathrm{pH} 5,0$ avant d'ajuster au $\mathrm{pH}$ de la mesure 
ne modifie que peu les temps de coagulation par rapport aux laits acidifiés directement (considérés comme témoins), sauf pour les échantillons à $\mathrm{pH}$ inférieur à 6,0 , dont le temps de coagulation est plus long.

L'addition de $0,23 \mathrm{mM} / 1$ de lysozyme entraîne une réduction du temps de coagulation à tous les $\mathrm{pH}$ étudiés (tab. 1). La figure 2-a montre que dans un lait à $\mathrm{pH} 6,0$, la réduction du temps de coagulation est d'autant plus importante que la quantité de lysozyme ajouté est grande, jusqu'à $0,3 \mathrm{mM} / 1$; l'addition d'une quantité plus importante est sans effet sur le temps de coagulation.

TABLEAU 1 - TABLE 1

Influence d'une acidification et d'une addition de lysozyme sur le temps de coagulation du lait

\begin{tabular}{|c|c|c|c|}
\hline \multirow{2}{*}{$\begin{array}{c}\mathrm{pH} \\
\mathrm{du} \text { lait }\end{array}$} & \multicolumn{3}{|c|}{$\begin{array}{l}\text { Temps de coagulation du lait (en secondes) } \\
\text { après différents traitements }\end{array}$} \\
\hline & Témoins & $\begin{array}{l}\text { Acidification } \\
\text { transitoire } \\
\text { à pH } 5,0\end{array}$ & $\begin{array}{l}\text { Addition de } 0,23 \mathrm{M} \\
\text { de lysozyme }\end{array}$ \\
\hline 5,0 & 195 & & 126 \\
\hline 5,3 & 218 & 325 & 144 \\
\hline 5,7 & 280 & 395 & 171 \\
\hline 6,0 & 454 & 456 & 281 \\
\hline 6,7 & 2185 & 2250 & 1042 \\
\hline 7,0 & 4800 & $>9000$ & 2250 \\
\hline 7,3 & $>9000$ & & 9000 \\
\hline \multirow{2}{*}{$\begin{array}{c}\text { Milk } \\
p H\end{array}$} & $\begin{array}{l}\text { Reference } \\
\text { samples }\end{array}$ & $\begin{array}{c}\text { Acidified to } \\
\text { pH 5,0 before final } \\
p H \text { adjustement }\end{array}$ & $\begin{array}{l}\text { Lysozyme } \\
\text { added }(0,23 \mathrm{mM})\end{array}$ \\
\hline & \multicolumn{3}{|c|}{$\begin{array}{c}\text { Rennet coagulation time of milks (seconds) } \\
\text { after different treatments }\end{array}$} \\
\hline
\end{tabular}




\section{DISCUSSION}

Nous avons montré que le pourcentage de CMP libéré au moment de la coagulation du lait par la présure est fonction du $\mathrm{pH}$. $\mathrm{Au} \mathrm{pH}$ naturel du lait $(\mathrm{pH}$ 6.7) la coagulation se produit à un taux d'hydrolyse du CMP de $92 \%$, valeur proche de celle qui avait été obtenue par Dalgleish (1979). Au pH 5,2, elle est seulement de $43 \%$ et il est probable que pour des $\mathrm{pH}$ inférieurs elle soit plus faible, mais nous n'avons pas réalisé l'expérimentation car l'acidification correcte du lait dans la masse posait des problèmes méthodologiques. L'effet d'un abaissement du $\mathrm{pH}$ sur la quantité de CMP libérée à la coagulation avait déjà été noté par Foltmann (1959). Ces résultats confirment l'importance de la charge micellaire dans le phénomène de coagulation. La libération du CMP, très chargé négativement (Green et Marshall, 1977), entraîne une diminution progressive de la charge micellaire, qui a été mise en évidence par la variation du potentiel $\mathrm{Z}$; il passe de $-17 \mathrm{mV}$ pour les micelles natives à $-10 \mathrm{mV}$ après action de la présure (Pearce, 1976). La coagulation du lait se produirait quand la charge micellaire atteint une " charge critique de déstabilisation ». La valeur de la charge micellaire baisse avec le pH du lait (Zittle et al., 1958) ainsi que celle du potentiel Z (Darling et Dickson, 1979). Selon le $\mathrm{pH}$, donc selon la charge micellaire, l'hydrolyse d'une quantité différente de CMP serait nécessaire pour atteindre la charge critique de déstabilisation et la coagulation.

Dans les expériences, où nous avons effectué une acidification transitoire du lait à pH 5,0 avant d'effectuer le test de coagulation, notre but était d'entraîner par ce traitement des changements structuraux dans la micelle et de modifier la répartition relative du phosphate de calcium et des caséines. La quantité totale de minéraux fixés est la même que dans les échantillons non pré-acidifiés au même pH (Fauquant, 1983), puisque cet équilibre ne dépend que du pH pour un lait de composition donnée (Mc Gann et Pyne, 1960). Pratiquement, nous avons observé une légère augmentation du calcium soluble, de l'ordre de $15 \%$ à tous les $\mathrm{pH}$ étudiés. L'augmentation de la force ionique due à l'addition de l'acide et de la base pourrait en être responsable. Nous avons montré que le traitement de pré-acidification à $\mathrm{pH} 5,0$ ne modifie pas la quantité de CMP hydrolysé au moment de la coagulation. Cela indique que l'organisation native du phosphate de calcium n'intervient pas dans le phénomène de coagulation, et confirme l'hypothèse d'une déstabilisation due à un équilibre de charges.

L'addition de lysozyme au lait entraîne une diminution du taux de CMP nécessaire à la coagulation. Plus le $\mathrm{pH}$ est bas, plus l'efficacité du lysozyme est grande, surtout si l'on tient compte du fait 
que sa fixation sur la micelle diminue avec le $\mathrm{pH}$. Aux $\mathrm{pH}$ supérieurs à 7,0 , il n'a plus d'influence sur le taux de CMP libéré à la coagulation. Ces résultats pourraient être expliqués par l'évolution de la charge du lysozyme qui diminue quand le $\mathrm{pH}$ augmente $(\mathrm{pH}$ isoélectrique 10,7). La diminution d'efficacité observée en fonction du $\mathrm{pH}$ semblerait alors confirmer qu'il intervient en modifiant la charge micellaire; il est possible également que les sites de fixation du lysozyme sur la micelle soient différents selon le $\mathrm{pH}$. Pour des teneurs en lysozyme de 0 à $0,3 \mathrm{mM} / 1$ nous avons observé que la diminution de la teneur en CMP libéré à la coagulation et le temps de coagulation sont inversement proportionnels à la quantité de lysozyme ajouté. La vitesse d'action enzymatique n'est donc pas modifiée par la présence de lysozyme. Green et Marshall (1977) avaient observé une augmentation de la vitesse de libération du NPN, surtout sensible en fin de réaction. Il n'y a pas contradiction entre ces résultats ; en effet, dans notre expérience, le $\mathrm{pH}$ du lait avait été ajusté à $\mathrm{pH}$ 6,0 et la coagulation se produisait donc alors que $75 \%$ seulement du CMP avait été libéré. En présence de quantités croissantes de lysozyme, elle se produit à des taux de 67 à $38 \%$ hydrolyse. A ces stades de la réaction, l'effet stimulant du lysozyme sur la vitesse de réaction n'est pas apparent.

La réduction du temps de coagulation observée après addition du lysozyme au lait est plus importante aux pH supérieurs à 6,0 $(-50 \%)$ qu'aux $\mathrm{pH}$ plus bas $(-35 \%)$. Nous avons mis en évidence que le taux de CMP à hydrolyser pour obtenir la coagulation s'élève avec le $\mathrm{pH}$, mais par ailleurs, on sait que l'élévation de $\mathrm{pH}$ ralentit la vitesse d'action de la présure, par une action directe (peut-être en relation avec une dénaturation de l'enzyme) et par l'intermédiaire de la diminution du calcium ionique libre (dont la teneur intervient également sur la vitesse de réaction). Les derniers stades de la réaction d'hydrolyse sont alors extrêmement lents et l'hydrolyse de la totalité du CMP requiert un temps très long. Quand on ajoute du lysozyme dans un lait, d'une part on abaisse légèrement le $\mathrm{pH}$, ce qui entraîne une augmentation de la vitesse de réaction, d'autre part, on provoque la coagulation à un taux de CMP libéré plus faible. La coagulation se produit avant que ne soient réalisés les derniers stades de la réaction : ils sont très lents à $\mathrm{pH}$ élevé, le temps de coagulation est donc fortement diminué; à un $\mathrm{pH}$ plus bas où les conditions d'hydrolyse sont plus favorables, la réduction du temps de coagulation est moindre.

\section{Remerciements}

Nous remercions G. Brulé pour d'utiles discussions, J. L. Maubois et J. Hermier pour la lecture critique du manuscrit. 


\section{Bibliographie}

DalgLeish (D. G.) (1979). - Proteolysis and aggregation of casein micelles treated with immobilized or soluble chymosin. Journal of Dairy Research, 46, 653-661.

Darling (D. F.) and Dickson (J.) (1979). - The determination of the Zeta potential of casein micelles. Journal of Dairy Research, 46, 329-332.

Davies (D. T.) and White (J.C. D.) (1960), - The use of ultrafiltration and dialysis in isolating the aqueous phase of milk and in determining the partition of milk constituents between the aqueous and disperse phases. Journal Dairy Research, 27, 171-190.

Fauquant (J.) (1983), - Communication personnelle.

Foltmann (B.) (1959). - On the enzymatic and the coagulation stages of the renneting process. XVe Congrès International de Laiterie de Londres, 3 - $a-21$, 655-661.

Mc Gann (T. C. A.) and Pyne (G. T.) (1960). - The colloidal phosphate of milk. III. Nature of its association with casein. Journal of Dairy Research, 27, 403-417.

GREen (M. L.) and CRUTCHField (G.) (1971). - Density-gradient electrophoresis of native and of rennet-treated casein micelles. Journal of Dairy Research, $38,151-164$.

Green (M. L.) and MARshall (R. J.) (1977). - The acceleration by cationic materials of the coagulation of casein micelles by rennet. Journal of Dairy Research, 44, 521-531.

GREEN (M. L.) (1982). - Effect on the composition and properties of casein micelles of interaction with ionic materials. Journal of Dairy Research, $49,87-98$.

Marshall (R. L.) and Green (M. L.) (1980). - The effect of chemical structure of additives on the coagulation of casein micelle suspensions by rennet. Journal of Dairy Research, 47, 359-369.

Noelken (M. E.) (1966). - Simulated $\alpha_{s_{1}}-$ K casein complex. Journal of Dairy Science, 49, 706 (note).

PAYENS (T. A. J.) (1966). - Association of caseins and their possible relation to structure of the casein micelles. Journal of Dairy Science, 49, 1317-1324.

PeARCE (K. N.) (1976). - Moving bondary electrophoresis of native and rennettreated casein micelles. Journal of Dairy Research, 43, 27-36.

Pyne (G. T.) and Mc Gann (T. C.A.) (1962), - The influence of the colloidal phosphate of milk on the rennet coagulation. XVI ${ }^{\mathrm{e}}$ Congrès International de Laiterie, B IV: 1, 611-616.

Schmidt (D. G.) (1980). - Colloidal aspects of casein. Netherland Milk and Dairy Journal, 34, 42-64.

SHugar (D. G.) (1952). - The measurement of lysozyme activity and the ultraviolet inactivation of lysozyme. Biochemica Biophysica Acta, 8, 302-309.

VAN Hooydonk (A. C. N.) and Olieman (C.) (1982). - A rapid and sensitive high performance liquid chromatography method of following the action of chymosin in milk. Netherland Milk Dairy Journal, 36, 153-158.

WAUGH (D. F.) and VoN HiPpel (P. H.) (1956). - K-casein and the stabilization of casein micelles. Journal of American Chemical Society, 78, 4576-4582.

Zittle (C. A.), Dellamonica (E. S.), Rudd (R. K.) and Custel (J. H.) (1958). Binding of calcium to casein: influence of $\mathrm{pH}$ and calcium and phosphate concentrations. Archives of Biochemistry and biophysics, 76, 342-353. 